\title{
Impact of low-dose calcipotriol ointment on wound healing, pruritus and pain in patients with dystrophic epidermolysis bullosa: A randomized, double-blind, placebo-controlled trial
}

Christina Guttmann-Gruber ${ }^{1 \dagger}$, Josefina Piñón Hofbauer ${ }^{1 \dagger}$, Birgit Tockner ${ }^{1}$, Victoria Reichl ${ }^{1}$, Alfred Klausegger ${ }^{1}$, Peter Hofbauer ${ }^{2}$, Martin Wolkersdorfer ${ }^{2}$, Khek-Chian Tham³ , Seong Soo Lim³ ${ }^{3}$ John E. Common³, Anja Diem ${ }^{4}$, Katharina Ude-Schoder ${ }^{4}$, Wolfgang Hitz ${ }^{5,6,7}$, Florian Lagler ${ }^{8}$, Julia Reichelt ${ }^{1}$, Johann W. Bauer ${ }^{1,9}$, Roland Lang ${ }^{9^{*}+}$ (I) and Martin Laimer ${ }^{1,9^{*}+}$

\begin{abstract}
Background: Wound management is a critical factor when treating patients with the inherited skin fragility disease dystrophic epidermolysis bullosa (DEB). Due to genetic defects in structural proteins, skin and mucous epithelia are prone to blistering and chronic wounding upon minor trauma. Furthermore, these wounds are commonly associated with excessive pruritus and predispose to the development of life-threatening squamous cell carcinomas, underscoring the unmet need for new therapeutic options to improve wound healing in this patient cohort. Vitamin D3 is acknowledged to play an important role in wound healing by modulating different cellular processes that impact epidermal homeostasis and immune responses. In this study, we evaluate the safety and efficacy of low-dose calcipotriol, a vitamin D3 analogue, in promoting wound healing and reducing itch and pain in patients with DEB.

Methods: Eligible DEB patients, aged $\geq 6$ years and with a known mutation in the COL7A1 gene, were recruited to a placebo-controlled, randomized, double blind, cross-over phase II monocentric clinical trial. Patients were required to have at least two wounds with a minimum size of $6 \mathrm{~cm}^{2}$ per wound. The primary objective was to evaluate efficacy of daily topical application of a $0.05 \mu \mathrm{g} / \mathrm{g}$ calcipotriol ointment in reducing wound size within a 4-week treatment regimen. Secondary objectives were to assess safety, as well as the impact of treatment on pruritus, pain, and bacterial wound colonization in these patients.
\end{abstract}

Results: Six patients completed the clinical trial and were included into the final analysis. Topical low-dose calcipotriol treatment led to a significant reduction in wound area at day 14 compared to placebo ( $88.4 \%$ vs. $65.5 \%, P<0.05)$. Patients also reported a significant reduction of pruritus with calcipotriol ointment compared to placebo over the

\footnotetext{
*Correspondence: r.lang@salk.at; m.laimer@salk.at

${ }^{\dagger}$ Christina Guttmann-Gruber, Josefina Piñón Hofbauer, Roland Lang, and

Martin Laimer have contributed equally to this work

${ }^{9}$ Department of Dermatology and Allergology, University Hospital

Salzburg, Paracelsus Medical University, Muellner-Hauptstrasse 48,

5020 Salzburg, Austria

Full list of author information is available at the end of the article
}

(c) The Author(s) 2021. Open Access This article is licensed under a Creative Commons Attribution 4.0 International License, which permits use, sharing, adaptation, distribution and reproduction in any medium or format, as long as you give appropriate credit to the original author(s) and the source, provide a link to the Creative Commons licence, and indicate if changes were made. The images or other third party material in this article are included in the article's Creative Commons licence, unless indicated otherwise in a credit line to the material. If material is not included in the article's Creative Commons licence and your intended use is not permitted by statutory regulation or exceeds the permitted use, you will need to obtain permission directly from the copyright holder. To view a copy of this licence, visit http://creativecommons.org/licenses/by/4.0/. The Creative Commons Public Domain Dedication waiver (http://creativeco mmons.org/publicdomain/zero/1.0/) applies to the data made available in this article, unless otherwise stated in a credit line to the data. 
entire course of the treatment as shown by itch scores of $3.16 \mathrm{vs} 4.83(P<0.05)$ and 1.83 vs $5.52(P<0.0001)$ at days 14 and 28 , respectively. Treatment with low-dose calcipotriol did not affect serum calcium levels and improved the species richness of the wound microbiome, albeit with no statistical significance.

Conclusions: Our results show that topical treatment with low-dose calcipotriol can accelerate wound closure and significantly reduces itch, and can be considered a safe and readily-available option to improve local wound care in DEB patients.

Trial Registration EudraCT: 2016-001,967-35. Registered 28 June 2016, https://www.clinicaltrialsregister.eu/ctr-search/ trial/2016-001967-35/AT

Keywords: Epidermolysis bullosa, Wound healing, Pruritus, Vitamin D3, Calcipotriol

\section{Background}

Vitamin D3 (VD3) is a hormone that is primarily known for its role in calcium homeostasis and bone metabolism. Moreover, it is a key regulator of various cellular pathways in different tissues regulating hormone secretion, cell proliferation and differentiation, and immune function [1]. In human skin, which acts as site for VD3 synthesis, it contributes to skin homeostasis and proper wound healing, engaging tissue repair mechanisms that enable keratinocytes to respond to injury and infection [2]. In this regard, VD3 influences both, the innate and adaptive immune system, by inducing the expression of anti-microbial peptides (AMPs) such as cathelicidin to elicit a first-line immune defense against microbes [3], as well as by modulating immune cell responses and inflammation [4], respectively. Proper function of these processes are particularly important for patients with the rare genetic skin disease epidermolysis bullosa (EB) who suffer from excessive tissue fragility due to mutations in structural genes important for skin integrity. As a consequence, blisters and wounds develop upon minor friction. One of the most severe forms is dystrophic EB (DEB) which is caused by homozygous or compound heterozygous mutations in the gene COL7A1, disrupting the formation of functional anchoring fibrils that tether the epidermis to the underlying dermis. Chronic wounding of the skin, and repeated cycles of skin regeneration, infection, inflammation and tissue remodeling subsequently promote the development of life-threatening squamous cell carcinoma (SCC) in this patient cohort [5]. VD3 is produced in the skin upon exposure to ultraviolet $\mathrm{B}$ (UBV) radiation but wound bandages and reduced outdoor activities limit epidermal VD3 production in affected EB individuals. Notably, VD3 deficiency $(<20 \mathrm{ng} / \mathrm{ml})$ is a common feature in recessive DEB despite oral supplementation [6].

In previous in vitro investigations, we demonstrated that an induction of the AMP cathelicidin, enhanced antimicrobial defense, and accelerated wound closure in a 2D cell culture model of recessive dystrophic EB (RDEB) using a low concentration $(100 \mathrm{nM})$ of the active VD3 analog calcipotriol [7]. The same treatment concentration further showed anti-neoplastic effects against established RDEB tumor cells, suggesting a safe application in this patient cohort who are highly prone to SCC development [8]. Importantly, significant clinical benefit in terms of enhanced wound closure was achieved in a single-patient observation study [7], wherein a significant reduction of itch was also reported. Together, these observations provided the rationale for evaluating the efficacy of a lowdose calcipotriol ointment in improving wound healing in RDEB patients in the present randomized, doubleblind, placebo-controlled phase II clinical trial (Fig. 1).

\section{Results \\ Patients}

In total, 12 DEB patients were enrolled into the study. However, three patients did not fulfill the inclusion criteria (two wounds $>6 \mathrm{~cm}^{2}$ ) and were excluded from the analysis. A further two patients could not enter the second intervention phase because they received additional medication prohibited by the protocol during the twomonth wash out phase. One patient was not included into the analysis because of incorrect follow-up of the target wounds. As a result, 6 patients were included into the final data analysis (see Flow chart, Additional file 1: Fig. 1).

\section{Efficacy of treatment on wound closure}

The clinical trial was designed as a cross-over study, wherein patients were randomized into one of two treatment arms in the first phase and, after a wash-out phase, crossed over into the other treatment arm in the second phase (Fig. 1). Accordingly, a patient who received placebo in the first phase crossed over into the calcipotriol group in the second phase, and viceversa. In total, twenty-four wounds (12 per intervention phase) were treated equally in a ratio of $1: 1$ with either calcipotriol ointment or placebo (Additional file 2: Table 1). Average wound sizes at the beginning of each treatment were $16.41 \mathrm{~cm}^{2}$ and $15.67 \mathrm{~cm}^{2}$ in the calcipotriol and placebo arms, respectively (Fig. 2a 


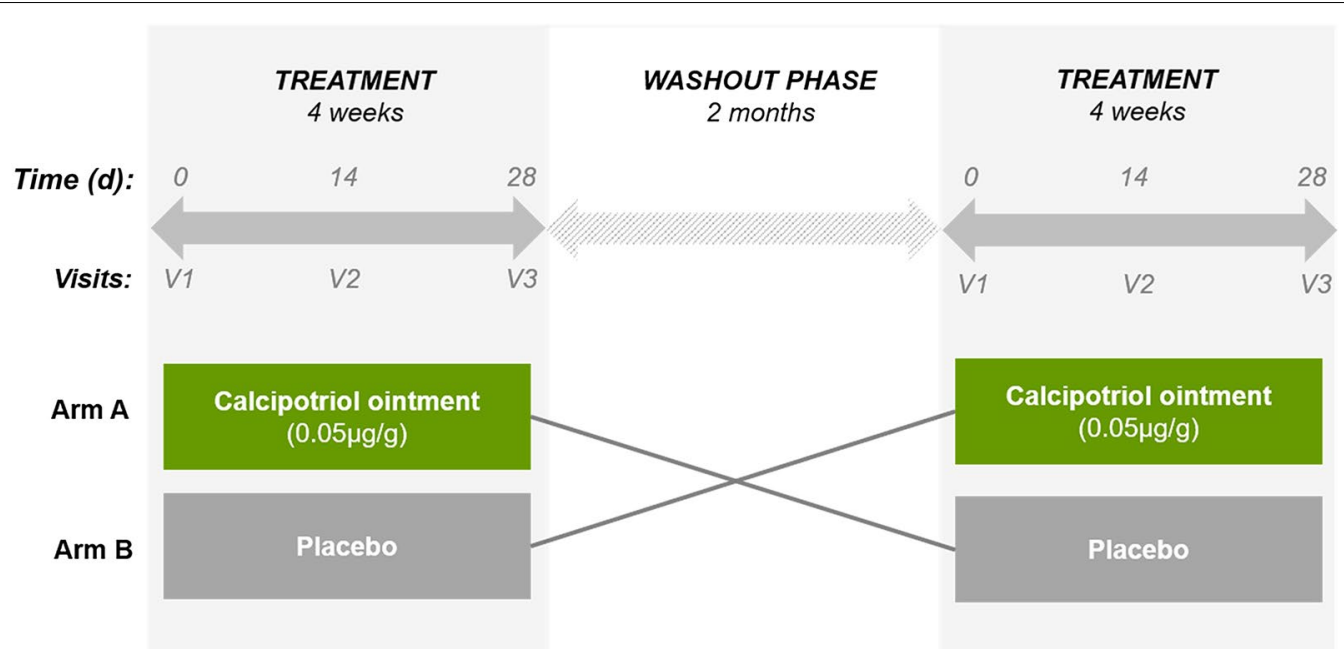

Fig. 1 Clinical study design. We performed a two-armed, double blind, randomized, cross-over phase II study. Patients were randomized into two groups receiving either calcipotriol ointment $(0.05 \mu \mathrm{g} / \mathrm{g})$ or the placebo. $1 \mathrm{~g}$ of ointment (either verum or placebo) was applied topically on each of two designated target wounds for a period of 4 weeks. After a 2-month wash-out phase the patient crossed over into the second treatment arm. Clinical assessment of patients was performed every two weeks (day 0, 14 and 28) at the study center when wounds were photographed and swabbed for microbiome analysis

and c). We observed a significant $88.4 \%$ reduction in wound size with calcipotriol treatment versus $65.6 \%$ with placebo $(P=0.006$; generalized estimation equation model) at day 14 . At that time point, calcipotriol reduced wound size to $<10 \%$ of baseline in $9 / 12$ wounds, as compared to $5 / 12$ which received the placebo. Both treatment and placebo resulted in the same number of wounds $(8 / 12)$ with sizes $<10 \%$ of baseline after 28 days (Fig. 2b). Notably the fraction of wounds that were completely closed was higher in the calcipotriol group compared to placebo although this did not reach statistical significance (7 vs $4,58.3 \%$ vs $33.3 \%$; Fisher's exact test, $P=0.4136$ ) (Additional file 3 : Table 2).

\section{Itch and pain}

Pruritus and pain rank high among reported patientrelevant complications associated with DEB [9]. Therefore, we assessed the impact of treatment on patient quality of life by evaluating itch and pain using a visual analog scale. Patients reported a significant and steady reduction in itch over the entire treatment course in the calcipotriol arm (Fig. 3a). No change in itch was recorded in the placebo arm. Both treatments resulted in reduced pain. While significantly improved pain scores were reported with placebo on day 14 (Fig. 3b), no significant differences between both treatment arms were observed at day 28 .

\section{Safety assessment}

No drug-related adverse reactions were observed with calcipotriol treatment. Two patients (P03 and P09) gave consent for blood sampling for evaluation of serum calcium levels to exclude hypercalcemia. Neither treatment affected serum calcium levels, which remained within the reference range of $2.13-2.63 \mathrm{mmol} / \mathrm{l}$ at the time points tested (Additional file 4: Table 3).

\section{Microbiome analysis}

We evaluated the impact of treatment on wound microbiota by shotgun whole-metagenomics sequencing from 5 patients (P03, P06, P08, P09, P12). Wounded areas showed reduced microbial diversity and increased abundance of Staphylococcus aureus compared to control intact skin (Fig. 4a and b), confirming previous observations [4, 5]. Three out of 5 patients showed increased species richness upon calcipotriol on day 14 compared to placebo (Fig. 4c), but no general effect on $S$. aureus abundance was observed (data not shown). Thus, while a tendency towards improved wound microbiome was observed in calcipotriol-treated closed wounds at day 14 and day 28 compared to placebo, this did not reach statistical significance (Fig. 4d).

\section{Discussion}

This trial suggests that topical application of calcipotriol $0.05 \mu \mathrm{g} / \mathrm{g}$ ointment is safe and well-tolerated by DEB patients. Topical Psorcutan ${ }^{\circledR}$ ointment (active 
A.

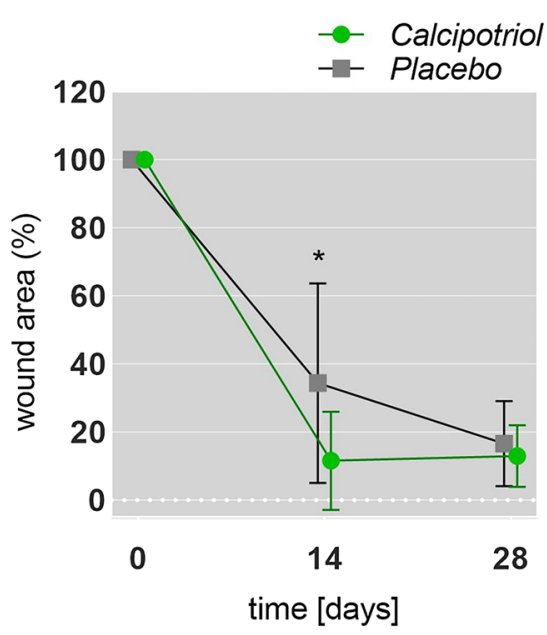

B.

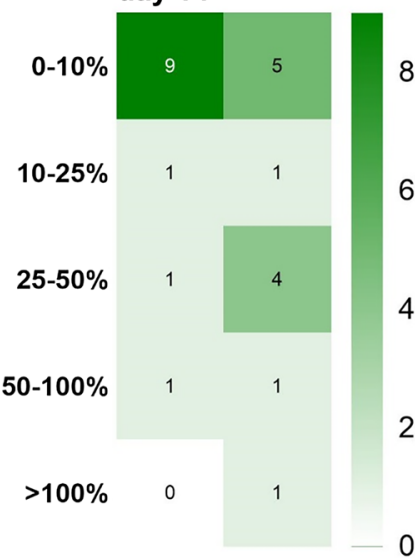

day 28

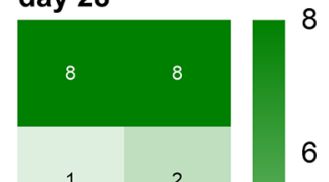

6

4

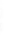

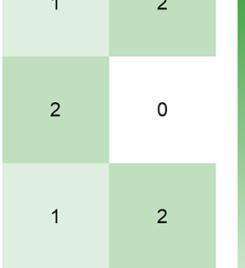

2

$0 \quad 0$

0

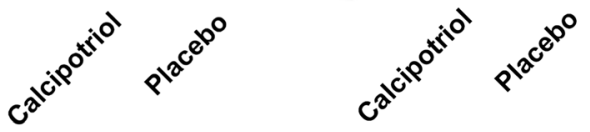

C.

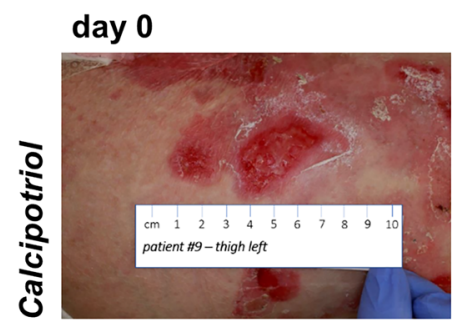

day 14

day 28
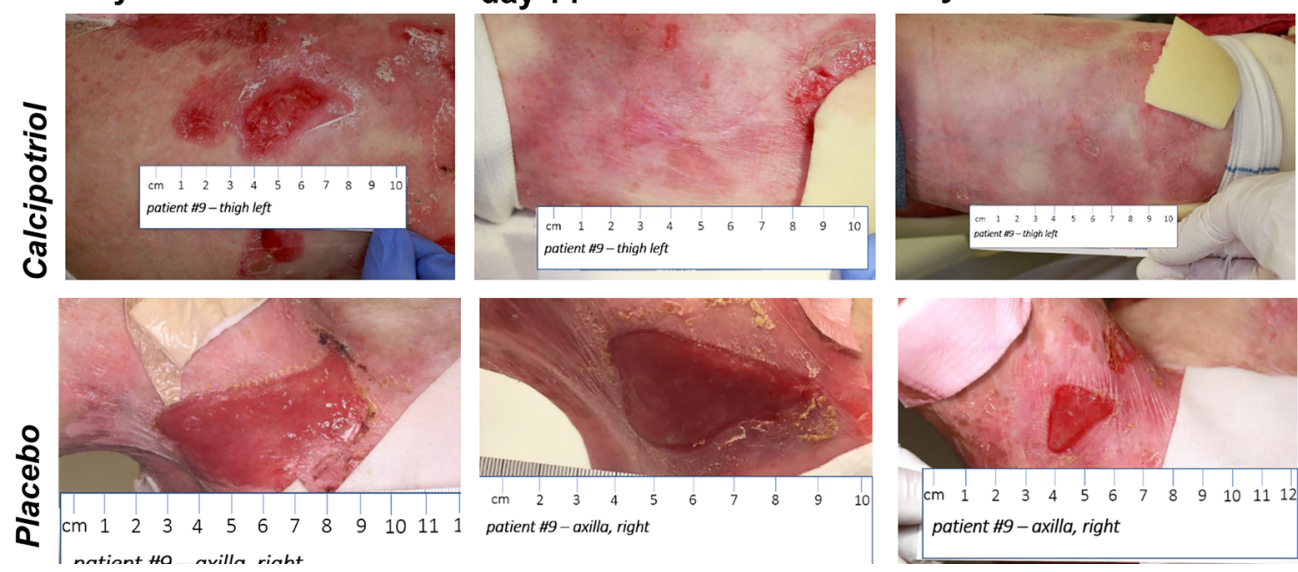

Fig. 2 Impact of low-dose calcipotriol treatment on wound healing. a Size of wound area (\%) representing mean with 95\% confidence intervals (Cls) at baseline (day 0), 14 and 28 days of treatment with low-dose calcipotriol or placebo. Statistical analysis; generalized estimation-equation model based on gamma distribution, ${ }^{*} P=0.006$. b Heat plot showing numbers of wounds with $0-10 \%, 10-20 \%, 25-50 \%, 50-100 \%$ or $>100 \%$ wound area compared to baseline after treatment with low-dose calcipotriol or placebo at 14 and 28 days. c Clinical pictures of wounds of a patient responding well to low-dose calcipotriol $(0.05 \mu \mathrm{g} / \mathrm{g})$ treatment at baseline, 14 and 28 days of treatment with low-dose calcipotriol or placebo

ingredient calcipotriol $50 \mu \mathrm{g} / \mathrm{g}$ ) is licensed for the treatment of psoriasis with an established safety profile for long-term use in adults and children ( $<6 \mathrm{yrs})$. Based on previous evidence, we applied a 1000 -fold less concentrated calcipotriol ointment onto the wounds of DEB patients in this present study. Both its established safety profile and the significantly reduced concentration of calcipotriol used, argued for the adequate safety standard of this approach. Nevertheless, we provide data to address safety concerns of potential systemic uptake of the drug owing to reduced skin barrier function in our patient cohort, and hypercalcemia due to VD3 regulation of calcium metabolism. Notably, monitoring for serum hypercalcemia subsequent to excessive calcipotriol uptake was restricted to study participants consenting to blood draws, reflecting the intention to limit invasive procedures on vulnerable skin in order to reduce the trial burden. By contrast, clinical symptoms of hypercalcemia (such as concentration disorder, personality change, adynamia, myasthenia, depression, dyspepsia, nausea, anorexia, constipation, polyuria, polydipsia, bone pain) were regularly assessed in all patients at each visit. Against this background, clinical monitoring remained inconspicuous in all patients 
A.
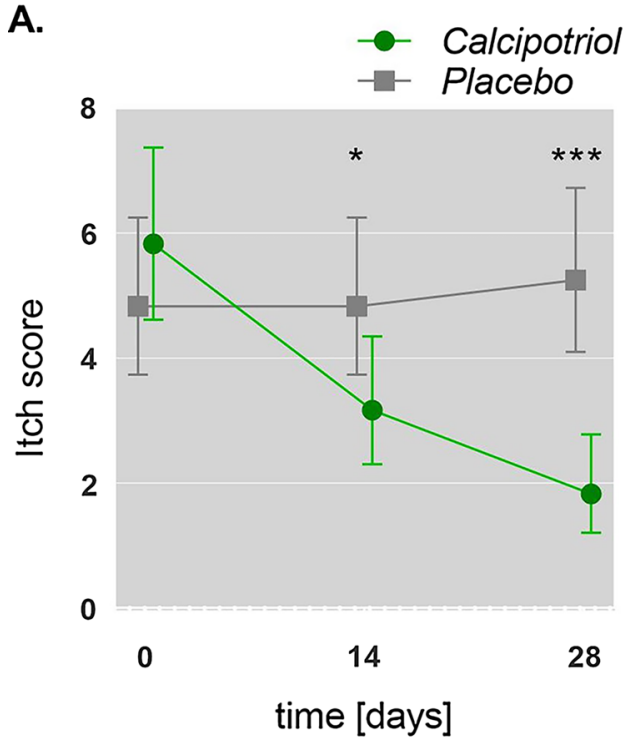

B.

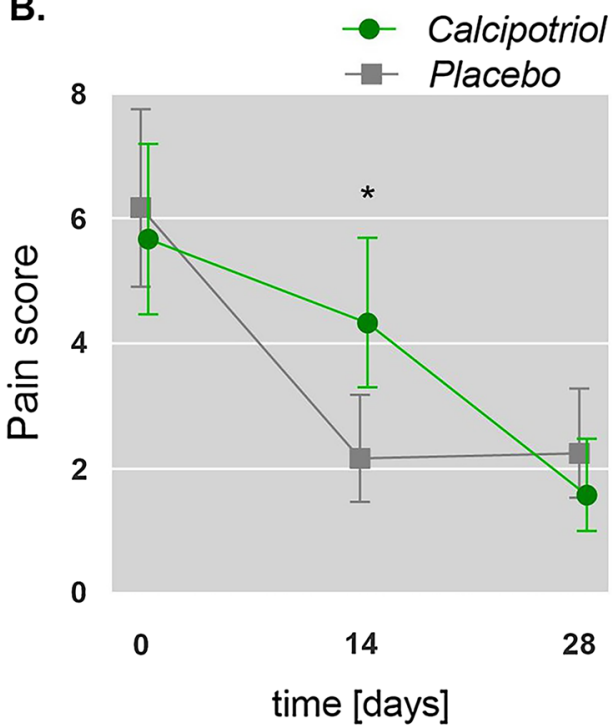

Fig. 3 Influence of low-dose calcipotriol treatment on itch (a) and pain (b). Scores were assessed for each wound at baseline (day 0), 14 and 28 days of treatment with low-dose calcipotriol or placebo using a visual analog scale (VAS) ranging from 0 (no itch/pain) to 10 (maximum itch/pain). Mean with $95 \%$ Confidence Intervals (Cls) are shown. Statistical analysis; generalized estimation-equation model based on gamma distribution, ${ }^{*} P<0.05$, ${ }^{* *} P<0.0001$

and laboratory analyses of two probands additionally showed no impact of treatment on serum calcium levels.

In psoriasis, calcipotriol is used to target keratinocyte dedifferentiation and hyperproliferation. Such an antiproliferative effect would be obviously detrimental in the setting of this trial, which is intended to foster wound healing in RDEB, a process that requires cell proliferation. However, our previous studies demonstrated that lower concentrations of calcipotriol have no impact on keratinocyte proliferation in vitro [7]. In line with these observations, our clinical data showed that wound closure was accelerated over placebo in $5 / 6$ patients after 2 weeks of treatment. Notably, accelerated wound closure in a greater fraction of wounds may translate into significant clinical benefit for patients. Accumulating evidence in mouse models highlight the interplay between microbial triggers, innate immune responses, and persistent skin inflammation in wounding-induced carcinogenesis, identifying critical molecular factors and immune cell subsets also shown to be relevant in dystrophic EB [10-12]. In this respect, the timely resolution of wounds potentially limits exposure of keratinocytes to these carcinogenic triggers.

Itch and pain rank highest among EB-associated morbidities that significantly decrease patient quality of life $[9,13]$. With regard to the analgesic potential of calcipotriol, our results remain inconclusive and elusive with patients reporting a statistically significant benefit at day
14 with placebo, but no difference between placebo and verum at day 28. However, oral substitution of D3 has been suggested to be beneficial for chronic pain management in individuals with VD3 deficiency [14]. A striking effect of calcipotriol over placebo was the reduction in itch, which was reported by $5 / 6$ patients in at least one wound and by $4 / 6$ patients in both wounds. The antipruritogenic effect of calcipotriol has been previously reported in clinical trials treating patients with psoriasis [15]. It can be partly attributed to the anti-inflammatory function of VD3 that stimulates $\mathrm{T}$ cells to reduce the expression of inflammatory cytokines e.g. TNF-alpha, IL-1, IL-2, IL-6 or IL-8 [4]. Notably, increased levels of inflammatory cytokines are detected in blister fluids and plasma from DEB patients as well [16-18]. Calcipotriol may also indirectly improve burden of itch by inducing the AMP cathelicidin (LL-37), which primarily acts in innate immune defense against microbial infection but in turn has also been shown to induce the nerve repulsion factor semaphorin 3A [19] thereby implicating a potential anti-pruritogenic effect. However, further investigations are warranted to decipher the molecular mechanisms underlying the anti-pruritogenic effects of low-dose calcipotriol in the EB skin.

The composition of the skin microbiome is essential for overall skin health contributing to tissue homeostasis and proper wound healing. Furthermore, skin commensals have been demonstrated to be important for controlling local inflammation and tuning resident $\mathrm{T}$ cell function 
A.

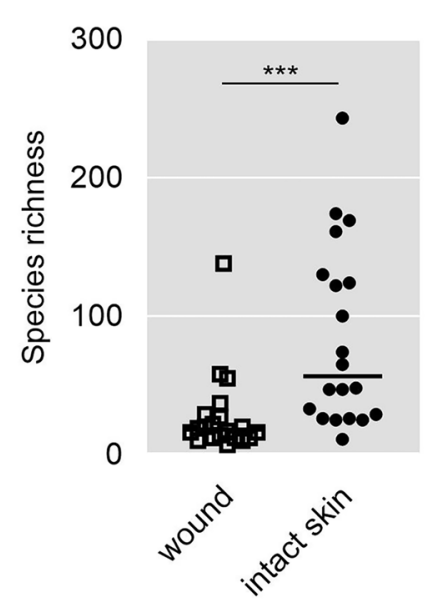

C.

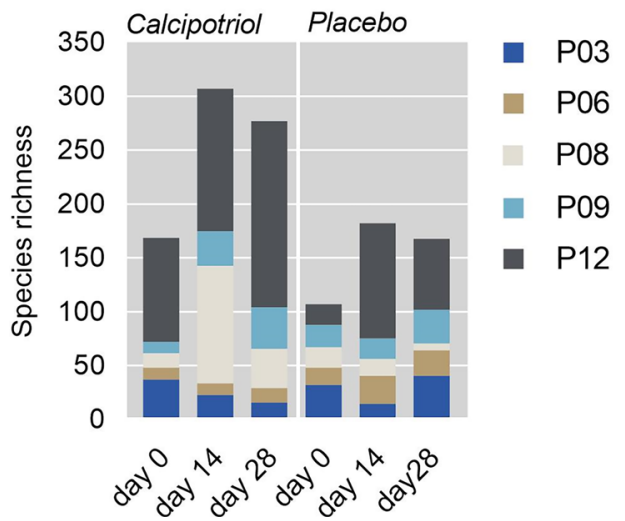

B.

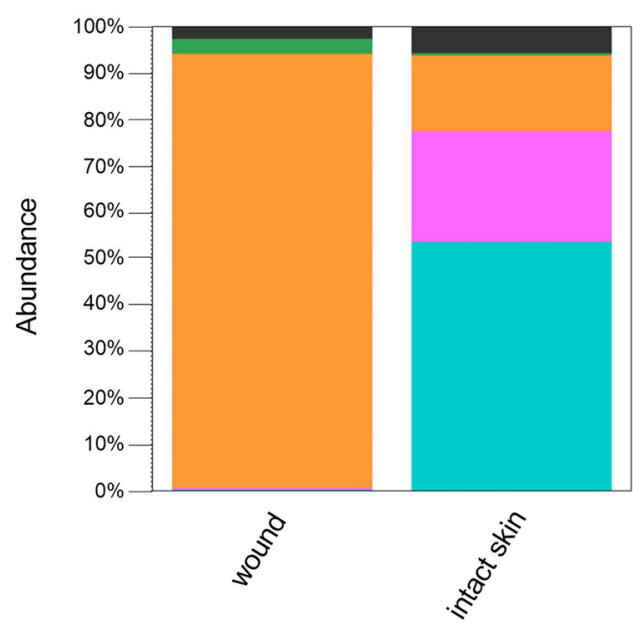

Other

Staphylococcus argenteus Staphylococcus aureus Staphylococcus epidermidis Staphylococcus caprae

D.

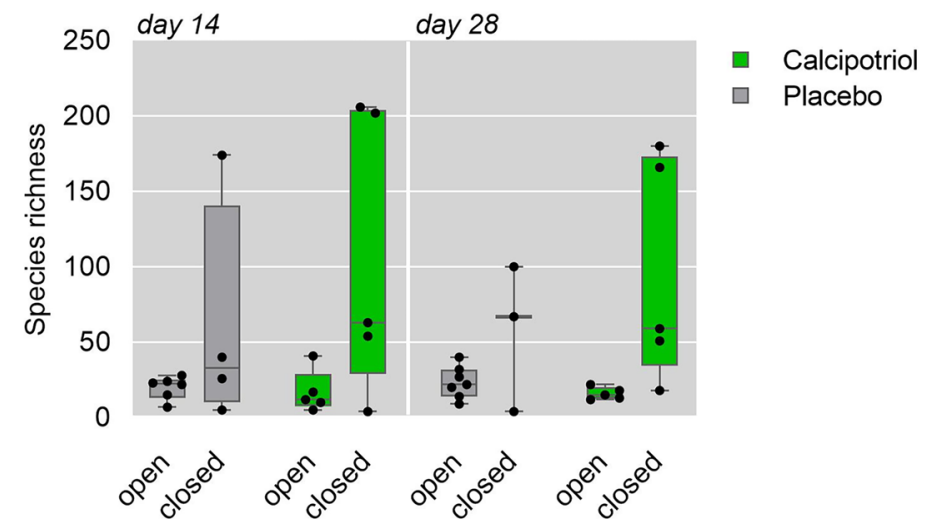

Fig. 4 Effects of low-dose calcipotriol treatment on wound microbiome. a Scatter plot showing species richness on intact skin compared to wounds. Each dot represents an individual wound at baseline (day 0). Statistical analysis; Mann Whitney test, ${ }^{* *} P=0.0001$. b Relative abundance of staphylococcus species in all wounds and on intact skin at baseline (day 0). c Microbial species richness of wounds at baseline, after 14 and 28 day-treatment with low-dose calcipotriol or placebo. Data are presented as mean of two wounds of individual patients. $\mathbf{d}$ Species richness in open vs completely closed wounds after 14 and 28 day- treatment with low-dose calcipotriol or placebo

in order to provide protective immunity against cutaneous pathogens [20]. This observation is highly relevant for RDEB patients since metagenomics analysis have revealed reduced microbial diversity with a corresponding increase in pathogenic species in skin and wounds in this patient cohort [21] suggesting a potential impaired immune function and wound healing due to dysbiosis. In this trial, we observed increased species richness in 3 out of 5 patients upon 2 weeks treatment with calcipotriol, suggesting a beneficial effect on microbial diversity. Considering its antimicrobial, immunomodulatory and wound healing potential, calcipotriol may thus interfere with key pathogenic traits in EB, including tumorigenesis, which supports its implementation into local wound management strategies.

A major limitation of this study is the low patient number owing to the rareness of the disease [22], which additionally precluded the stratification of acute and chronic wounds to identify those that would benefit most from treatment. The study time points, initially chosen based on reports of EB wounds remaining open for $>4$ weeks [23], appear to inadequately reflect the potency of topical low-dose calcipotriol on wound closure. Protocol-based intensified wound care may have additionally enhanced the effects of calcipotriol and placebo treatment over standard of care, potentially contributing to overall accelerated wound closure in this study compared to published literature. Thus, the primary objective, i.e. a $40 \%$ reduction of wound area with calcipotriol compared to placebo after 4 weeks treatment was not reached. In this context, our data indicate that assessment at earlier time points $(<14$ days) is warranted. Time-to-wound-closure may additionally be a more relevant endpoint to comprehensively determine 
the therapeutic impact of calcipotriol treatment. A dosefinding study, which so far could not be performed due to limited patient numbers, may further aid in determining the optimal dose for wound healing and its impact on natural disease course.

\section{Conclusions}

While corroboration of our results by large-scaled studies is pending, our preliminary data suggest that topical low-dose calcipotriol ointment significantly reduces itch, accelerates wound healing, and can be safely implemented into the daily wound care of DEB patients.

\section{Methods and study design Patient cohort}

In total, we enrolled 12 RDEB patients with a diagnosed COL7A1 gene mutation, of which 9 completed both intervention phases. 6 patients were included into the final analysis (Additional file 1: Fig. 1 and Additional file 2: Table 1). All patients gave written informed consent to participate in the trial. Exclusion criteria included pregnancy, breast feeding, participation in other clinical trials, known impaired kidney or liver dysfunction, known disorders of calcium metabolism, or systemic treatment with corticosteroids, immunosuppressive drugs or antibiotics.

\section{Study medication}

Psorcutan ${ }^{\circledR}$ ointment containing $50 \mu \mathrm{g} / \mathrm{g}$ calcipotriol (LEO Pharma, Vienna, Austria) was diluted 1:1000 in Ultraphil ${ }^{\circledR}$ (Bayer, Vienna, Austria), reaching a therapeutic concentration of $0.05 \mu \mathrm{g} / \mathrm{g}(\sim 121 \mathrm{nM})$ calcipotriol as assessed in our single-patient observation [7]. Ultraphil ${ }^{\circledR}$ base alone served as placebo control. Production and blinding of study medication was performed by the Good Manufacturing Practice (GMP)-certified drug production department of the hospital pharmacy according to EU GMP guidelines.

\section{Treatment}

Patients were randomized into two treatment arms and instructed to apply $1 \mathrm{~g}$ of either verum- (active ingredient: calcipotriol $0.05 \mu \mathrm{g} / \mathrm{g}$ ) or placebo-containing ointment topically onto two designated target wounds $\left(\geq 6 \mathrm{~cm}^{2}\right.$ each) daily over a period of four weeks. No other changes to standard of care of the patients were applied. Thus, while patients were instructed not to apply any other topical substances onto the designated target wounds, all other EB lesions were continued to be concurrently treated with the previous local wound dressings.

After a two-month wash out-phase, patients crossed over into the other treatment arm. Wounds were photographed, measured and swabbed for microbiome profiling at study visits (V) on day 0 (V1/V4), 14 (V2/V5) and 28 (V3/V6) during each treatment period.

Blood drawing was optional for the patient and was performed at the beginning and end of each treatment phase to assess serum calcium levels.

\section{Wound area measurement}

Wound area was assessed by overlaying a transparent gridded foil onto the wound and tracing the wound edges with a permanent pen. Drawings were scanned and wound area was calculated (in $\mathrm{cm}^{2}$ ) by three independent, blinded investigators using ImageJ software (Additional file 4: Table 3). Statistical analysis was performed on mean wound area measurements. Pruritus and pain scores were assessed using a visual analog scale ranging from 0 (no itch/pain) to 10 (maximum itch/pain) at every study visit.

\section{Microbiome sampling and data analysis}

Sampling. Sterile cotton swabs (Catch-All Sample collection Swab) were pre-moistened in sterile SCF-1 buffer (50 mM Tris buffer [pH 7.6], 1 mM EDTA [pH 8], 0.5\% Tween 20), manufactured by the Hospital Pharmacy, Salzburg, Department of Production and immediately applied to the body site. Each target wound and the corresponding intact skin on the opposite site of the body was sampled according the Essener Kreisl technique [24] at each study visit. The head of the swab was cut off with sterile scissors, placed into sterile tubes containing SCF-1 buffer and stored at $-80^{\circ} \mathrm{C}$ until further processing.

\section{DNA extraction, library construction and sequencing}

Swabs were transferred into Lysing Matrix E tubes (MP Biomedicals) together with $100 \mu \mathrm{L}$ of ATL Buffer (Qiagen) containing 1.2\% Triton X-100. Next, $10 \mu \mathrm{l}$ of lysozyme $(50 \mathrm{mg} / \mathrm{ml}$, \#90,082, Thermo Scientific) were added and incubated at $37^{\circ} \mathrm{C}$ for $30 \mathrm{~min}$. Samples were then subjected to bead-beating (FastPrep-24, MP Biomedicals) at $6.0 \mathrm{~m} / \mathrm{s}$ for $40 \mathrm{~s}$. Samples were centrifuged at $16,000 \times \mathrm{g}$ for $5 \mathrm{~min}$. Supernatants were then treated with $10 \mu \mathrm{l}$ Proteinase K (Qiagen) and incubated at $56^{\circ} \mathrm{C}$ for $15 \mathrm{~min}$. DNA was extracted (EZ1 Advanced XL Instrument, Qiagen) using the EZ1 DNA Tissue Kit (Qiagen) and quantified with Qubit dsDNA HS Assay Kit (Life Technologies) and stored at $-20{ }^{\circ} \mathrm{C}$ prior to downstream use. The extracted DNA samples were used to construct libraries using NEBNext Ultra II FS (New England Biolabs) and barcoded adaptors according to the manufacturer's E7805 protocol. Custom indexed primers with 14 PCR cycles were used for enrichment of DNA libraries, which were quantified using Agilent DNA 1000 Kit (Agilent Technologies) and Agilent 2100 Bioanalyzer (Agilent Technologies). Samples were normalized and pooled for 
paired-end sequencing $(2 \times 150 \mathrm{bp})$ using Illumina HiSeq $\mathrm{X}$ platform.

\section{Bioinformatics analysis}

The raw reads in fastq format were imported into the commercial CLC Genomics Workbench 12.0.3 using the default setting: Illumina high-throughput sequencing import, paired reads, paired-end (forward-reverse) with a minimum distance of 1 and a maximum distance of 1000 . Using the default workflow of data QC and taxonomic profiling, the trimming quality score limit was set at 0.05 , a trim adapter list containing Illumina adapter sequences (first read: AGATCGGAAGAGCACACGTCTGAA CTCCAGTCA; second read: AGATCGGAAGAGCGT CGTGTAGGGAAAGAGTGT) for 3' end trimming was added, a CLC curated reference database that contains 979 archaea sequences and 34,866 bacteria sequences (version June 2019 - 22 GB) was used for taxonomic mapping and the hg38 genome reference was used for host read filtering.

\section{Statistical analysis}

Data were checked for consistency and normality. Generalized estimating equation models based on gamma and Poisson distributions were used. Least significant difference tests were used for pairwise comparisons. All reported tests were two-sided, and $P$ values $<0.05$ were considered statistically significant. All statistical analyses were performed by using PASW 24 (IBM SPSS Statistics for Windows, Version 21.0, Armonk, NY) and GraphPad Prism (GraphPad Software, Inc.,Version 9.0, San Diego, CA).

\section{Abbreviations \\ EB: Epidermolysis bullosa; DEB: Dystrophic EB; RDEB: Recessive DEB; V: Visit; VAS: Visual Analog Scale; VD3: Vitamin D3.}

\section{Supplementary Information}

The online version contains supplementary material available at https://doi. org/10.1186/s13023-021-02062-2.

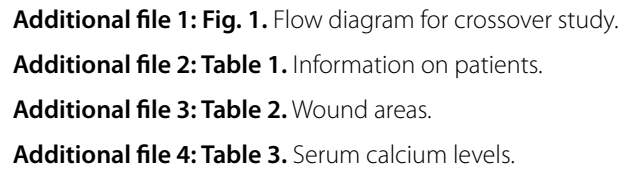

\section{Acknowledgements}

We would like to acknowledge LEO Pharma for providing Psorcutan ${ }^{\circledR}$ ointment and charge certificate. Finally, we thank EB patients and their legal guardians for taking part in the clinical trial and DEBRA Austria for funding. This work was generated within the European Reference Network (ERN)-Skin. All co-authors state no conflict of interest or any financial interest relevant to the subject of the manuscript.

\section{Authors' contributions}

$\mathrm{ML}$ (principal investigator) and RL (study coordinator) had full access to all data in the study and take responsibility for the integrity of the data and the accuracy of the data analysis. $M L, R L, C G G, J P H$ provided concept and designed the study. JEC, KCT, SSL, ML, AD, KUS, BT, VLR, RL, CGG and JPH assisted with acquisition, analysis and interpretation of the data. WH performed statistical analysis. ML, RL, CGG and JPH drafted the manuscript. MW, PH, BT, AK, VLR, JR and FL provided administrative, technical or material support. RL, ML and JWB supervised the clinical trial. All authors critically revised the manuscript for important intellectual content. All authors gave final approval of the submitted manuscript.

\section{Funding}

The work was funded by DEBRA Austria, a non-profit patient organization.

\section{Availability of data and materials}

The dataset used and/or analyzed during the current study are available from the corresponding author on reasonable request.

\section{Declarations}

\section{Ethics approval}

This placebo-controlled, randomized, double-blind, cross-over, phase II clinical trial (EudraCT: 2016-001967-35) was approved by the ethics committee of Salzburg (415-E/2043/7-2016).

\section{Consent for publication}

Patients or their legal guardians gave written informed consent for publication of the data generated in this trial.

\section{Competing interests}

The authors declare that they have no competing interests.

\section{Author details}

${ }^{1}$ Present Address: EB House Austria, Research Program for Molecular Therapy of Genodermatoses, Department of Dermatology and Allergology, University Hospital of the Paracelsus Medical University, Salzburg, Austria. ${ }^{2}$ Landesapotheke Salzburg, Department of Production, Hospital Pharmacy, Salzburg, Austria. ${ }^{3}$ Skin Research Institute of Singapore, A*STAR, 8A Biomedical Grove, Immunos \#06-06, Singapore, Singapore. ${ }^{4}$ EB House Austria, Outpatient Unit, Department of Dermatology and Allergology, Paracelsus Medical University, Salzburg, Austria. ${ }^{5}$ Research Office Biostatistics, Paracelsus Medical University, Salzburg, Austria. ${ }^{6}$ Department of Ophthalmology and Optometry, Paracelsus Medical University Salzburg, Müllner Hauptstr. 48, 5020 Salzburg, Austria. ${ }^{7}$ Research Program Experimental Ophthalmology and Glaucoma Research, Paracelsus Medical University, Muellner Hauptstrasse 48, 5020 Salzburg, Austria. ${ }^{8}$ Institute for Inborn Errors of Metabolism and Department of Pediatrics, Paracelsus Medical University, Salzburg, Austria. ${ }^{9}$ Department of Dermatology and Allergology, University Hospital Salzburg, Paracelsus Medical University, Muellner-Hauptstrasse 48, 5020 Salzburg, Austria.

Received: 17 May 2021 Accepted: 30 September 2021

Published online: 08 November 2021

\section{References}

1. Bikle D. Vitamin D: Production, metabolism, and mechanisms of action. In: Feingold KR, Anawalt B, Boyce A, Chrousos G, de Herder WW, Dhatariya K, et al., editors. Endotext. South Dartmouth (MA) 2000.

2. Bikle D, Christakos S. New aspects of vitamin D metabolism and action - addressing the skin as source and target. Nat Rev Endocrinol. 2020;16(4):234-52.

3. Gombart AF, Borregaard N, Koeffler HP. Human cathelicidin antimicrobial peptide (CAMP) gene is a direct target of the vitamin D receptor and is strongly up-regulated in myeloid cells by 1,25-dihydroxyvitamin D3. FASEB J. 2005;19(9):1067-77.

4. Colotta F, Jansson B, Bonelli F. Modulation of inflammatory and immune responses by vitamin D. J Autoimmun. 2017;85:78-97. 
5. Kim M, Li M, Intong-Wheeler LRA, Tran K, Marucci D, Murrell DF. Epidemiology and outcome of squamous cell carcinoma in epidermolysis bullosa in Australia and New Zealand. Acta Derm Venereol. 2018;98(1):70-6.

6. Reimer A, Hess M, Schwieger-Briel A, Kiritsi D, Schauer F, Schumann $\mathrm{H}$, et al. Natural history of growth and anaemia in children with epidermolysis bullosa: a retrospective cohort study. Br J Dermatol. 2020;182(6):1437-48.

7. Guttmann-Gruber C, Tockner B, Scharler C, Huttner C, Common JE, Tay ASL, et al. Low-dose calcipotriol can elicit wound closure, anti-microbial, and anti-neoplastic effects in epidermolysis bullosa keratinocytes. Sci Rep. 2018;8(1):13430.

8. Robertson SJ, Orrin E, Lakhan MK, O'Sullivan G, Felton J, Robson A, et al. Cutaneous Squamous Cell Carcinoma in Epidermolysis Bullosa: a 28-year Retrospective Study. Acta Derm Venereol. 2021.

9. Bruckner AL, Losow M, Wisk J, Patel N, Reha A, Lagast H, et al. The challenges of living with and managing epidermolysis bullosa: insights from patients and caregivers. Orphanet J Rare Dis. 2020;15(1):1.

10. Fuentes I, Guttmann-Gruber C, Tockner B, Diem A, Klausegger A, CofreAraneda $G$, et al. Cells from discarded dressings differentiate chronic from acute wounds in patients with Epidermolysis Bullosa. Sci Rep. 2020;10(1):15064.

11. Hoste E, Arwert EN, Lal R, South AP, Salas-Alanis JC, Murrell DF, et al. Innate sensing of microbial products promotes wound-induced skin cancer. Nat Commun. 2015;6:5932.

12. Hoste E, Maueroder C, vanHove L, Catrysse L, Vikkula HK, Sze M, et al. Epithelial HMGB1 Delays Skin Wound Healing and Drives Tumor Initiation by Priming Neutrophils for NET Formation. Cell Rep. 2019;29(9):2689-701 e4.

13. Tang JY, Marinkovich MP, Lucas E, Gorell E, Chiou A, Lu Y, et al. A systematic literature review of the disease burden in patients with recessive dystrophic epidermolysis bullosa. Orphanet J Rare Dis. 2021;16(1):175.

14. Helde-Frankling M, Bjorkhem-Bergman L. Vitamin D in Pain Management. Int J Mol Sci. 2017;18(10).

15. Takahashi H, Tsuji H, Ishida-Yamamoto A, lizuka H. Comparison of clinical effects of psoriasis treatment regimens among calcipotriol alone, narrowband ultraviolet B phototherapy alone, combination of calcipotriol and narrowband ultraviolet B phototherapy once a week, and combination of calcipotriol and narrowband ultraviolet B phototherapy more than twice a week. J Dermatol. 2013;40(6):424-7.

16. Alexeev V, Salas-Alanis JC, Palisson F, Mukhtarzada L, Fortuna G, Uitto J, et al. Pro-Inflammatory Chemokines and Cytokines Dominate the
Blister Fluid Molecular Signature in Patients with Epidermolysis Bullosa and Affect Leukocyte and Stem Cell Migration. J Invest Dermatol. 2017:137(11):2298-308

17. Annicchiarico G, Morgese MG, Esposito S, Lopalco G, Lattarulo M, Tampoia M, et al. Proinflammatory Cytokines and Antiskin Autoantibodies in Patients With Inherited Epidermolysis Bullosa. Medicine (Baltimore). 2015;94(42):e1528.

18. Lettner T, Lang R, Bauer JW, Wally V. Increased levels of matrix metalloproteinase- 9 and interleukin- 8 in blister fluids of dystrophic and junctional epidermolysis bullosa patients. J Eur Acad Dermatol Venereol. 2015:29(2):396-8.

19. Umehara Y, Kamata Y, Tominaga M, Niyonsaba F, Ogawa H, Takamori K. Cathelicidin LL-37 Induces Semaphorin 3A Expression in Human Epidermal Keratinocytes: Implications for Possible Application to Pruritus. J Invest Dermatol. 2015;135(11):2887-90.

20. Naik S, Bouladoux N, Wilhelm C, Molloy MJ, Salcedo R, Kastenmuller W, et al. Compartmentalized control of skin immunity by resident commensals. Science. 2012;337(6098):1115-9.

21. Fuentes I, Guttmann-Gruber C, Tay ASL, Pinon Hofbauer J, Denil S, Reichelt J, et al. Reduced microbial diversity is a feature of recessive dystrophic epidermolysis bullosa-involved skin and wounds. J Invest Dermatol. 2018;138(11):2492-5.

22. Fine JD. Epidemiology of inherited epidermolysis bullosa based on incidence and prevalence estimates from the national epidermolysis bullosa registry. JAMA Dermatol. 2016;152(11):1231-8.

23. Petrof G, Martinez-Queipo M, Mellerio JE, Kemp P, McGrath JA. Fibroblast cell therapy enhances initial healing in recessive dystrophic epidermolysis bullosa wounds: results of a randomized, vehicle-controlled trial. Br J Dermatol. 2013;169(5):1025-33.

24. Al Ghazal P, Korber A, Klode J, Schmid EN, Buer J, Dissemond J. Evaluation of the Essen Rotary as a new technique for bacterial swabs: results of a prospective controlled clinical investigation in 50 patients with chronic leg ulcers. Int Wound J. 2014;11(1):44-9.

\section{Publisher's Note}

Springer Nature remains neutral with regard to jurisdictional claims in published maps and institutional affiliations.
Ready to submit your research? Choose BMC and benefit from:

- fast, convenient online submission

- thorough peer review by experienced researchers in your field

- rapid publication on acceptance

- support for research data, including large and complex data types

- gold Open Access which fosters wider collaboration and increased citations

- maximum visibility for your research: over $100 \mathrm{M}$ website views per year

At BMC, research is always in progress.

Learn more biomedcentral.com/submissions 